\title{
Electrolyte and protein imbalance following anti-EGFR therapy in cancer patients: A comparative study
}

\author{
PIUS MALIAKAL and ANDREA LEDFORD \\ M.D. Anderson Cancer Center Orlando, Orlando, FL 32806, USA
}

Received November 23, 2009; Accepted December 24, 2009

DOI: $10.3892 /$ etm_00000047

\begin{abstract}
Electrolytic changes in blood have been associated with the use of anti-epidermal growth factor receptor (EGFR) antibodies. The objective of this retrospective study was to compare the rate of incidence and the severity of blood electrolyte and protein imbalances occurring in patients receiving cetuximab or panitumumab with or without concurrent chemotherapy. Treatment data of 58 patients who received cetuximab and 21 patients who received panitumumab were analyzed. Cetuximab caused hypomagnesemia in more than half of the patients, among whom 4 had severity up to grade 2/3 level, whereas panitumumab induced hypomagnesemia in $90 \%$ of the patients with severity up to grade 2 level occurring in $38 \%$ of the patients. Intravenous magnesium supplementation on the day of anti-EGFR treatment did not always adequately control the magnesium wasting in these patients. Often treatments with these agents had to be interrupted or terminated as a result of severe electrolyte depletion despite supplementation. Taking into consideration the mechanism of magnesium wasting from the kidney and the magnesium transport process in the gut, intravenous magnesium infusion coupled with oral supplementation with more tolerant oral magnesium products may help improve the treatment outcome in these patients. Surprisingly, more than half of these patients showed significant decreases in their albumin levels, which were correlated with the initiation or discontinuation of anti-EGFR therapy. The underlying mechanism of this decrease in albumin level is not known. The increased likelihood of poor outcomes such as mortality, morbidity and prolonged hospital stay in acutely ill patients with hypoalbuminemia is well recognized. Moreover, the maintenance of adequate serum albumin levels in these patients receiving anti-EGFR therapy may play an important role in containing some of the adverse effects of concurrently administered chemotherapeutic agents.
\end{abstract}

Correspondence to: Dr Pius Maliakal, M.D. Anderson Cancer Center Orlando, 1400 South Orange Ave, Orlando, FL 32806, USA E-mail: pius.maliakal@orlandohealth.com

Key words: epidermal growth factor receptor inhibitors, electrolytic changes, cetuximab, panitumumab, hypomagnesemia, hypocalcemia, hypoalbuminemia

\section{Introduction}

The epidermal growth factor receptor (EGFR) is a member of the erbB family of four related cell membrane receptors, including EGFR (HER1 or erbB1), HER2 (erbB2), HER3 (erbB3) and HER4 (erbB4). These receptors possess an extracellular $\mathrm{N}$-terminal ligand-binding domain, a hydrophobic transmembrane domain involved in interactions between receptors within the cell membrane, and a C-terminal intracellular domain and multiple phosphorylation sites. EGFR can be activated through a series of events initiated by binding to its ligands, such as EGF, TGF- $\alpha$, amphiregulin, epiregulin and hemiregulin. By binding to its ligands, EGFR triggers the homodimerization of EGFR or heterodimerization of EGFR with other ErbBs, resulting in the autophosphorylation of the receptor. The activated receptor recruits signaling complexes and activates the MEK, ERK, PI-3-K, STATS and PLC- $\gamma$ pathways (1). These pathways are potent oncogenic regulators of tumor cell growth, invasion, angiogenesis and metastasis. EGFR is overexpressed in many human epithelial carcinomas including colorectal, esophageal, prostate, ovarian, head and neck, lung and kidney, and this overexpression may play a role in tumorigenesis and poor patient prognosis and outcome (2).

Principal strategies for EGFR target therapy include the monoclonal antibody (mAb) and small molecule tyrosine kinase inhibitors. mAb blocks the receptor extracellular domain inhibiting ligand binding and receptor activation, and the small molecule tyrosine kinase inhibitors do not permit autophosphorylation by blocking the intracellular domain. Cetuximab (Erbitux) and panitumumab (Vectibix) are monoclonal antibodies which bind specifically to EGFR on both normal and tumor cells, and competitively inhibit the binding of ligands leading to the inhibition of cell proliferation, differentiation and survival.

Cetuximab is a chimeric $\mathrm{mAb}$ which normally contains approximately $34 \%$ of murine proteins (3). It was first approved in 2004 for colorectal cancer and later for head and neck cancers. Chimeric mAb is less immunogenic than murine $\mathrm{mAb}$ and has improved therapeutic utility. However, due to their murine sequences, both may cause allergic reactions in a portion of the patients $(4,5)$. Panitumumab is a fully humanized $\mathrm{mAb}$ and was approved for the treatment of EGFR-expressing metastatic colorectal carcinoma (6). Fully humanized mAbs are normally non-immunogenic and thus 
allow repeated administration without a human anti-human antibody response (7).

Occasional hypomagnesemia, followed by secondary hypokalemia, and hypocalcemia have been reported as side effects of cetuximab and panitumumab. Soon after the approval of cetuximab for colorectal cancer treatment, Schrag et al originally reported the occurrence and clinical course of hypomagnesemia in 34 of 154 patients who were treated with cetuximab in their institution (8). Out of 154 patients, 10 had grade 3 or 4 hypomagnesemia. Hypomagnesemia was also reported in $38 \%$ patients treated with panitumumab, with grade 3 and 4 hypomagnesemia occurring in $3 \%$ of those treated with panitumumab (9). Fakih et al, in a retrospective study, reported the incidence of hypomagnesemia in $27 \%$ of patients treated with cetuximab. Recently, Tejpar et al prospectively measured magnesium concentrations in a cohort of 98 patients treated with EGFR-targeting antibodies with or without combined chemotherapy (10). Ninety-seven percent of the patients had decreasing serum magnesium concentrations during EGFR-targeting treatment compared to baseline measurements. The mean serum magnesium slope during EGFR-targeting treatment (with or without combined chemotherapy) was significantly lower compared to chemotherapy alone.

In the present study, we aimed to retrospectively assess the occurrence of electrolyte depletion in patients treated with cetuximab or panitumumab with or without combined chemotherapy. Moreover, we aimed to compare the electrolytic changes caused by these two agents as well as to track any noticeable changes in other routine laboratory parameters in these patients.

\section{Materials and methods}

A study proposal was made to the Institutional Review Board of M.D. Anderson Cancer Center Orlando, and a waiver of authorization was obtained before initiating the study. Data from records of all patients who received treatment with cetuximab or panitumumab at the M.D. Anderson Cancer Center Orlando between January 1, 2007 and January 1, 2008 were reviewed.

The data collection method consisted of reviewing the records of the patients identified from the two patient database systems used in this institution (Sunrise XA and HBOC data base). Patient-specific data were securely de-identified and entered into a master key log for analysis. The parameters collected included the dose, route and date of drug administered, and various laboratory parameters including hematology and blood chemistry. Statistical analysis was performed using SPSS $^{\circledR}$.

\section{Results}

During the study period, 58 patients received treatment with cetuximab, with or without combined chemotherapy, of which approximately $1 / 3$ had head and neck cancer; the rest were treated for colorectal cancer. Twenty-one patients received panitumumab; all had colorectal cancer. The treatment duration spanned from just over 2 weeks to more than 1 year.

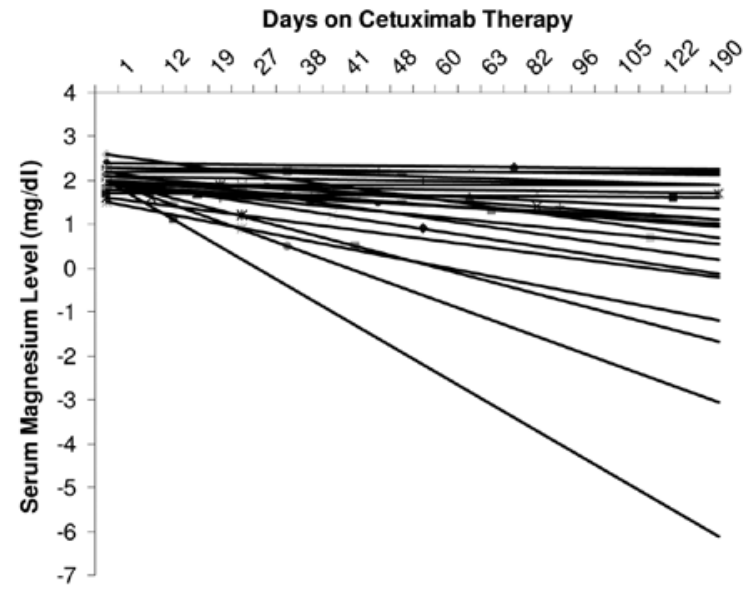

Figure 1. Changes in serum magnesium after commencing cetuximab therapy in patients. Each data point represents the baseline serum magnesium and the lowest serum magnesium levels during the treatment period. Straight lines connecting the data points represent a downward trend in the serum magnesium level changes.

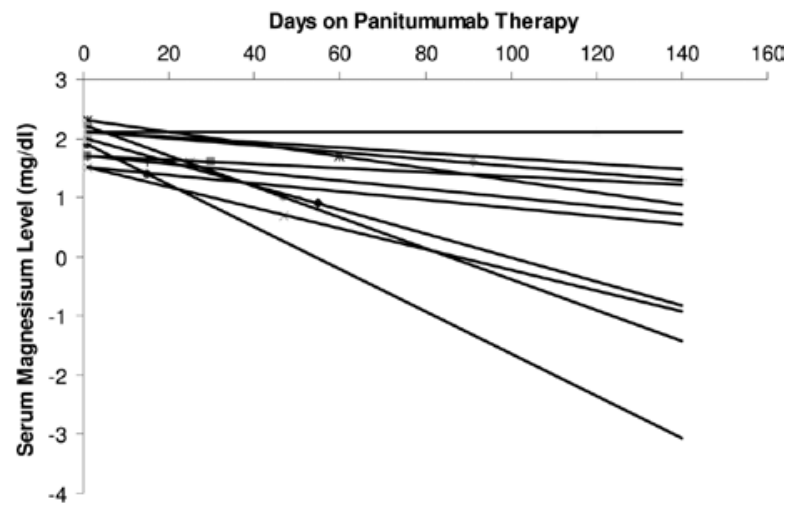

Figure 2. Changes in serum magnesium after commencing panitumumab therapy in patients. Each data point represents the baseline serum magnesium and the lowest serum magnesium levels during the treatment period. Straight lines connecting the data points represent a downward trend in the serum magnesium level changes.

Of the 58 patients who received cetuximab, 32 experienced varying grades of hypomagnesemia during the treatment period. Of these patients, 28 had grade 1 hypomagnesemia $(<1.7-1.2 \mathrm{mg} / \mathrm{dl}), 3 \mathrm{had}$ grade $2(<1.2-0.9 \mathrm{mg} / \mathrm{dl})$ and $1 \mathrm{had}$ grade 3 hypomagnesemia $(<0.9-0.7 \mathrm{mg} / \mathrm{dl})$. The slopes of the linear trend lines connecting the serum magnesium levels in these patients were almost exclusively negative (Fig. 1). There were few patients whose magnesium levels were not monitored. Thirty-eight patients showed some grades of decrease in blood calcium level, of which 5 had grade 2 and the remaining grade 1 hypocalcemia.

Of the 21 patients who received panitumumab, 18 had a decrease in their serum magnesium levels to varying extents. The slopes of the linear trend lines connecting the serum magnesium levels in these patients were almost exclusively negative (Fig. 2). The serum magnesium level was not monitored in 1 patient. Ten patients had grade 1 and 8 patients had grade 2 hypomagnesemia. Table I documents the comparison of hypomagnesemia and hypocalcemia incidence rates in 
Table I. Comparison of the rate of incidence of hypomagnesemia and hypocalcemia in patients who received cetuximab vs. panitumumab.

\begin{tabular}{|c|c|c|c|c|c|c|}
\hline \multirow[t]{2}{*}{ Anti-EGFR antibody } & \multicolumn{3}{|c|}{ Hypomagnesemia incidence (\%) } & \multicolumn{3}{|c|}{ Hypocalcemia incidence (\%) } \\
\hline & Grade $1^{\mathrm{a}}$ & Grade $2^{b}$ & Grade $3 / 4^{c}$ & Grade $1^{\mathrm{d}}$ & Grade $2^{\mathrm{e}}$ & Grade $3 / 4^{\mathrm{f}}$ \\
\hline Cetuximab & 48 & 5 & 2 & 56 & 10 & 0 \\
\hline Panitumumab & 52 & 38 & 0 & 52 & 14 & 0 \\
\hline
\end{tabular}

${ }^{\mathrm{a}}<1.7-1.2 \mathrm{mg} / \mathrm{dl} ;{ }^{\mathrm{b}}<1.2-0.9 \mathrm{mg} / \mathrm{dl} ;{ }^{\mathrm{c}}<0.9 \mathrm{mg} / \mathrm{dl} ;{ }^{\mathrm{d}}<8.9-8 \mathrm{mg} / \mathrm{dl} ;{ }^{\mathrm{e}}<8-7 \mathrm{mg} / \mathrm{dl} ;{ }^{\mathrm{f}}<7 \mathrm{mg} / \mathrm{dl}$.

Table II. Comparison of the rate of incidence of hypoalbuminemia in patients who received cetuximab vs. panitumumab.

\begin{tabular}{lccc}
\hline $\begin{array}{l}\text { Anti-EGFR } \\
\text { antibody }\end{array}$ & \multicolumn{3}{c}{$\begin{array}{c}\text { Hypoalbuminemia } \\
\text { incidence (\%) }\end{array}$} \\
\cline { 2 - 4 } & ${\text { Grade } 1^{\mathrm{a}}}^{\mathrm{a}}$ & Grade 2 & Grade 3c \\
\hline Cetuximab & 22 & 36 & 3 \\
Panitumumab & 19 & 42 & 7 \\
\hline
\end{tabular}

${ }^{\mathrm{a}}<3.5-3 \mathrm{~g} / \mathrm{dl} ;{ }^{\mathrm{b}}<3-2 \mathrm{~g} / \mathrm{dl} ;{ }^{\mathrm{c}}<2 \mathrm{~g} / \mathrm{dl}$.

patients who received cetuximab vs. the patients who received panitumumab.

The serum potassium level was also affected in many of the patients who received both these anti-EGFR agents. Surprisingly, in all the patients there was a considerable decrease in the serum albumin levels. Initially it was thought that these changes in the albumin levels were related to the disease itself. However, careful observation showed a direct relationship of the changes in albumin with the initiation or discontinuation of the treatments with the two anti-EGFR antibodies. Thirty-six patients who received cetuximab had varying severity of hypoalbuminemia during anti-EGFR treatment. Among them 13 patients had grade 1, 21 had grade 2, and 2 had grade 3 hypoalbuminemia.

Patients who received panitumumab also exhibited a similar pattern of hypoalbuminemia, with 15 out of 21 patients showing different grades. Of the 15 patients, 9 had grade 2 and 2 had grade 3 hypoalbuminemia; the rest had grade 1 . Table II compares the hypoalbuminemia noted in patients receiving both agents.

Seventeen patients required magnesium or/and calcium supplementation during cetuximab therapy. Magnesium and calcium supplementation was given as intravenous administration on the day of the cetuximab therapy. However, in many of these patients the serum magnesium levels continued to plunge before they were due for the next dose of cetuximab. A higher proportion of patients (15 patients) on panitumumab had received magnesium and/or calcium supplementation during the treatment period, given intravenously on the day of treatment.

\section{Discussion}

In this study, the serum levels of magnesium, calcium and albumin were monitored in patients who received the anti-EGFR antibodies cetuximab and panitumumab. Since cetuximab was approved for use in colorectal and head and neck cancers, there have been a few studies, both prospective and retrospective, investigating the decrease in the serum magnesium levels as an adverse effect. There have been no studies comparing the changes in blood electrolytes between populations of patients taking cetuximab and panitumumab.

The result of this retrospective study shows an approximate $60 \%$ rate of incidence of hypomagnesemia (grade 1-4) in patients receiving cetuximab, which is comparable to previously reported studies (11). We noted that the incidence of hypomagnesemia was higher in the case of patients treated with panitumumab compared to cetuximab. The severity of hypomagnesemia was also greater in patients receiving panitumumab. However, it should be noted that the sample sizes of the patients receiving these two agents were different. Continuous monitoring of magnesium levels was not mandated in some of the clinical trials involving cetuximab, nor in the standard care practices of some institutions $(12,13)$. The mandatory monitoring of serum magnesium levels would have revealed more cases of hypomagnesemia in these patients.

Magnesium is critically important in maintaining normal cell function, and symptomatic magnesium depletion is often associated with multiple biochemical abnormalities including hypokalemia, hypocalcemia and metabolic acidosis. As a result, hypomagnesemia is sometimes difficult to attribute solely to specific clinical manifestations. Severe hypomagnesemia is a serious electrolyte abnormality, the clinical manifestations of which include neuromuscular, cardiovascular and metabolic manifestations.

The earliest symptoms of magnesium deficiency are usually neuromuscular and neuropsychiatric disturbances, the most common being hyperexcitability. Neuromuscular irritability including tremor, fasciculation, tetany, Chvostek and Trousseau signs and convulsions were reported when hypomagnesemia was induced in volunteers. Other manifestations include convulsions, apathy, muscle cramps, hyperflexia, acute organic brain syndromes, depression, generalized weakness, anorexia and vomiting. The cardiovascular effects of magnesium deficiency include effects on electrical activity (prolonged QT and QU interval), myocardial contractility (ventricular 
tachycardia, Torsade de pointes, ventricular fibrillation), potentiation of digitalis effects and vascular tone.

Metabolic manifestations may include hypokalemia and hypocalcemia. Hypokalemia is a common event in patients with hypomagnesemia, occurring in $40-60 \%$ of cases. This is partly due to the underlying disorders that cause magnesium and potassium loss, including diuretic therapy or diarrhea.

The classic sign of severe hypomagnesemia $(<1.2 \mathrm{mg} / \mathrm{dl})$ is hypocalcemia. The mechanism is multifactorial. Parathyroid gland function is abnormal, largely due to impaired release of PTH. Impaired magnesium-dependent adenyl cyclase generation of cAMP mediates the decreased release of PTH. Skeletal resistance to this hormone in magnesium deficiency has also been implicated. Hypomagnesemia also alters the normal heteroionic exchange of calcium and magnesium at the bone surface, leading to an increased bone release of magnesium ions in exchange for an increased skeletal uptake of calcium from the serum.

The details of the exact mechanism of hypomagnesemia in patients receiving anti-EGFR antibodies remain unclear. However, recently, there have been some insights on the matter. In healthy individuals, serum magnesium concentrations are tightly controlled and vary between 0.70 and $1.1 \mathrm{mmol} / \mathrm{l}$ (14). In the kidney, approximately $80 \%$ of the serum magnesium is ultrafiltered in the glomeruli. Approximately $15-20 \%$ is reabsorbed in the proximal tubule. Subsequently, $70 \%$ of the magnesium is reabsorbed passively by a paracellular transport process in the thick ascending loop (TAL) driven by lumenpositive transepithelial voltage. However, the fine tuning of magnesium excretion takes place in the distal convoluted tubule (DCT), where 5-10\% of the filtered magnesium is reabsorbed via an active transcellular transport process (15). Apical entry into DCT cells is mediated by the magnesium permeable channel TRPM6 (transient receptor potential cation channel, subfamily $\mathrm{M}$, member 6) driven by a favorable transmembrane voltage (16). Magnesium entry into DCT cells appears to be the rate limiting step and site of regulation. Finally, 3-5\% of the filtered magnesium is excreted in the urine (14). TRPM6 is also expressed in intestinal epithelia where it plays a role in magnesium uptake. In the kidney, EGFR was detected in glomerular epithelial cells, TAL and DCT (17). Thus, both EGFR and TRPM6 are predominantly expressed in the DCT, the main site of active renal $\mathrm{Mg}^{2+}$ reabsorption. Moreover, it has been shown that the EGF dose dependently stimulated TRPM6 activity (18), and cetuximab significantly inhibited the activity of TRPM6 in human embryonic kidney cells (19).

The management of anti-EGFR antibody-inducing hypomagnesemia has always been challenging. In most patients the toxicity has been grade 1 or $2(0.9 \mathrm{mg} / \mathrm{dl}$ to lower limit of normal), grade $3(0.7-0.8 \mathrm{mg} / \mathrm{dl})$ or grade 4 ( $\leq 0.6 \mathrm{mg}$ / dl). Mostly grade 1 hypomagnesemia patients are generally asymptomatic and do not require magnesium supplementation. In patients with higher grade hypomagnesemia, intravenous replacement (2-3 g of magnesium sulfate) was administered mostly on the day of the anti-EGFR therapy. This method of replenishment was not adequate in some patients, in particular those with grade 3 hypomagnesemia. Many patients who have higher grade hypomangnesemia often present symptoms of fatigue, cramps or sleepiness. Patients who receive a combination of chemotherapy and anti-EGFR antibodies do not normally complain about these symptoms because they tend to attribute them to cytotoxic chemotherapy. It has been shown that the reversal of hypomagnesemia in patients of this category resulted in improvements in energy level and performance status (11). The management of hypomagnesemia needs to be aggressive in patients with grade 3 or 4 hypomagnesemia. Therefore, very high doses of magnesium are needed to achieve clinically significant reversal of these symptoms. Weekly magnesium replacement is often inadequate and serum magnesium levels fall back to low baseline level within 3-4 days. There have been reports suggesting that these patients would require daily to twice weekly treatment with intravenous magnesium to a level up to 6-10 g/ dose. There have also been cases of continuing or worsening grade 4 hypomagnesemia despite the daily $10 \mathrm{~g}$ of magnesium replacement (11). The possible reason for this is that as long as the EGFR in the kidney and intestine is inhibited, the renal magnesium wasting coupled with inadequate intestinal absorption will worsen. Since EGFR inhibition is rapid and long lasting after anti-EGFR therapy, the effectiveness of IV $\mathrm{Mg}^{2+}$ supplementation diminishes. As long as the EGFR inhibition continues in the body, magnesium wasting from the body is perpetuated. Thus, the alleviation of symptoms is transient and the deficient symptoms recur soon before the next dose of EGFR inhibitor. Since these agents are either given as weekly or bi-weekly cycles, the management of hypopmagnesemia and secondary hypocalcemia becomes difficult and sometimes the therapy needs to be interupted or terminated. Moreover, intravenous $\mathrm{Mg}^{2+}$ loading in these patients causes a significant renal $\mathrm{Mg}^{2+}$ leak. A possible way to control this would be through the use of oral magnesium supplements. In oral magnesium transport, TRPM6 plays a role in its absorption in the gut. Also, evidence suggests that autosomal recessive hypomagnesemia with secondary hypocalcemia (HSH) is caused by a mutation in TRPM6, which encodes the TRPM6 protein (20). In HSH patients, substitution of high oral doses of magnesium achieved good serum magnesium levels (14). However, diarrhea and other gastrointestinal disturbances associated with the use of magnesium supplementation, often limits its importance in treating many patients receiving antiEGFR therapy. The selection of better tolerated magnesium supplements would benefit these patients.

Observation of hypoalbuminemia was unexpected, as there has been no documentation of this as an adverse effect of anti-EGFR antibody therapy. Initially, it was thought that this change was related to the disease itself rather than caused by the anti-EGFR therapy. Surprisingly in this patient population, there appeared to be a good correlation between the initiation/discontinuation of the therapy and the changes in albumin level. The normal serum concentration of albumin in healthy adults is approximately 35-50 g/l. Hypoalbuminemia is common in seriously ill patients. The increased likelihood of poor patient outcomes such as mortality, morbidity and prolonged hospital stay in acutely ill patients with hypoalbuminema is well recognized. Because of its importance as an outcome predictor, serum albumin level was included as one of the component parameters in the Acute Physiology and Chronic Health Evaluation (APACHE) score (21). The medical literature documents several examples of an inverse relation- 
ship between serum albumin levels and survival in patients with advanced cancer (22). Many studies, spanning 40 years, have demonstrated that subclinical cancer, particularly of the breast, cervix and ovaries, may occur in more than $60 \%$ of pregnant women, when albumin remains between 20-25 g/l, and spontaneous remission normally is achieved postpartum when albumin returns to more than $43 \mathrm{~g} / \mathrm{l}$ (23). It is well recognized that the restoration of albumin profiles usually leads to full remission. When the albumin level falls it causes relapse. This is clearly demonstrated in Hodgkin's disease (24).

Serum albumin plays a diverse, complex and important role in maintaining physiological homeostasis. At reduced albumin levels, these homeostatic functions may be impaired, resulting in the development and/or progression of pathologic processes underlying poor outcome. Albumin has an antioxidant and free radical scavenging activity, a capacity to prevent apoptosis and an affinity for binding lipids, drugs, toxic substances and other ligands (25). Because of its high frequency in a broad range of pathologic conditions, hypoalbuminemia may plausibly be interpreted as a normal compensatory mechanism not requiring intervention. For instance, albumin redistribution into the interstitial space may provide protection from oxidative stress affecting extravascular tissues during disease states.

The mechanism of hypoalbuminemia in patients receiving anti-EGFR therapy is unknown. Plasma volume expansion due to overhydration, external albumin losses resulting from albuminuria, malnutrition, defective albumin synthesis, catabolism and distribution are some of the possible reasons for a decrease in serum albumin levels. The primary cause of low albumin in cancer patients has been identified by some researchers $(26,27)$ as the specific inhibition of albumin gene transcription by the tumor necrosis factor (TNF).

Many chemotherapy agents, in particular those with high lipid solubility, bind extensively to albumin. It is also important to note that the relative concentrations of free or albumin-bound fractions of a chemotherapeutic agent will influence the severity of its side effects. Therefore, the maintenance of adequate serum albumin levels in patients receiving anti-EGFR therapy would play a key role in containing some of the adverse effects of concurrently administered chemotherapeutic agents.

In the present study, serum levels of magnesium, calcium and albumin in patients receiving cetuximab and panitumumab were compared. The rate of incidence of hypomagnesemia was comparable to previously reported studies, but was found to be higher in patients treated with panitumumab compared to cetuximab. The severity of hypomagnesemia was also greater in patients receiving panitumumab. The observation of hypoalbuminemia was an unexpected adverse effect of the anti-EGFR antibody therapy. However, the inclusion of case-matched controls in the study would have added more value to the data.

\section{References}

1. Hynes NE and Lane HA: ERBB receptors and cancer: the complexity of targeted inhibitors. Nat Rev Cancer 5: 341-354, 2005.

2. Di Fiore PP, Pierce JH, Fleming TP, et al: Overexpression of the human EGF receptor confers an EGF-dependent transformed phenotype to NIH 3T3 cells. Cell 51: 1063-1070, 1987.
3. Riechmann L, Clark M, Waldmann $\mathrm{H}$ and Winter G: Reshaping human antibodies for therapy. Nature 332: 323-327, 1988.

4. Baselga J, Pfister D, Cooper MR, et al: Phase I studies of antiepidermal growth factor receptor chimeric antibody C225 alone and in combination with cisplatin. J Clin Oncol 18: 904-914, 2000.

5. Robert F, Ezekiel MP, Spencer SA, et al: Phase I study of anti-epidermal growth factor receptor antibody cetuximab in combination with radiation therapy in patients with advanced head and neck cancer. J Clin Oncol 19: 3234-3243, 2001.

6. Peeters M, Balfour J and Arnold D: Review article: panitumumab - a fully human anti-EGFR monoclonal antibody for treatment of metastatic colorectal cancer. Aliment Pharmacol Ther 28: 269-281, 2008.

7. Lynch DH and Yang XD: Therapeutic potential of ABX-EGF: a fully human anti-epidermal growth factor receptor monoclonal antibody for cancer treatment. Semin Oncol 29: 47-50, 2002.

8. Schrag D, Chung KY, Flombaum C and Saltz L: Cetuximab therapy and symptomatic hypomagnesemia. J Natl Cancer Inst 97: 1221-1224, 2005.

9. Van Cutsem E, Siena S, Humblet Y, et al: An open-label, single-arm study assessing safety and efficacy of panitumumab in patients with metastatic colorectal cancer refractory to standard chemotherapy. Ann Oncol 19: 92-98, 2008.

10. Tejpar S, Piessevaux H, Claes K, et al: Magnesium wasting associated with epidermal-growth-factor receptor-targeting antibodies in colorectal cancer: a prospective study. Lancet Oncol 8: 387-394, 2007.

11. Fakih M: Management of anti-EGFR-targeting monoclonal antibody-induced hypomagnesemia. Oncology 22: 74-76, 2008.

12. Sobrero A, Fehrenbacher L and Rivera F: Randomized Phase III trial of cetuximab plus irinotecan versus irinotecan alone for metastatic colorectal cancer in 1298 patients who have failed prior oxaliplatin-based therapy. Program and abstracts of the American Association for Cancer Research Annual Meeting, Los Angeles, April 2007.

13. Van Cutsem E, Nowacki M and Lang I: Randomized Phase III study of irinotecan and 5-FU/FA with or without cetuximab in the first-line treatment of patients with metastatic colorectal cancer (mCRC): the CRYSTAL trial. Program and abstracts of the American Association for Cancer Research Annual Meeting, Los Angeles, April 2007.

14. Konrad M, Schlingmann KP and Gudermann T: Insights into the molecular nature of magnesium homeostasis. Am J Physiol Renal Physiol 286: F599-F605, 2004.

15. Hoenderop JG and Bindels RJ: Epithelial $\mathrm{Ca}^{2+}$ and $\mathrm{Mg}^{2+}$ channels in health and disease. J Am Soc Nephrol 16: 15-26, 2005.

16. Voets T, Nilius B, Hoefs S, et al: TRPM6 forms the $\mathrm{Mg}^{2+}$ influx channel involved in intestinal and renal $\mathrm{Mg}^{2+}$ absorption. J Biol Chem 279: 19-25, 2004.

17. Gesualdo L, Di Paolo S, Calabro A, et al: Expression of epidermal growth factor and its receptor in normal and diseased human kidney: an immunohistochemical and in situ hybridization study. Kidney Int 49: 656-665, 1996.

18. Sack $\mathrm{E}$ and Talor Z: High affinity binding sites for epidermal growth factor (EGF) in renal membranes. Biochem Biophys Res Commun 154: 312-317, 1988.

19. Groenestege WM, Thebault S, van der Wijst J, et al: Impaired basolateral sorting of pro-EGF causes isolated recessive renal hypomagnesemia. J Clin Invest 117: 2260-2267, 2007.

20. Schlingmann KP, Weber S, Peters M, et al: Hypomagnesemia with secondary hypocalcemia is caused by mutations in TRPM6, a new member of the TRPM gene family. Nat Genet 31: 166-170, 2002.

21. Knaus WA, Wagner DP, Draper EA, et al: The APACHE III prognostic system. Risk prediction of hospital mortality for critically ill hospitalized adults. Chest 100: 1619-1636, 1991.

22. Lis CG, Grutsch JF, Vashi PG and Lammersfeld CA: Is serum albumin an independent predictor of survival in patients with breast cancer? JPEN J Parenter Enteral Nutr 27: 10-15, 2003.

23. Seaton K: Albumin, cancer and pregnancy. J Natl Med Assoc 94: 629-630, 2002.

24. Gobbi PG, Gendarini A, Crema A, et al: Serum albumin in Hodgkin's disease. Cancer 55: 389-393, 1985.

25. Emerson TE Jr: Unique features of albumin: a brief review. Crit Care Med 17: 690-694, 1989.

26. Peters T: All About Albumin. New York Academic Press, New York, 1996.

27. Seaton K: Albumin concentration controls cancer. J Natl Med Assoc 93: 490-493, 2001. 